\title{
ON THE OPTIMAL CONTROL COMPUTATION OF LINEAR SYSTEMS
}

\author{
H. Tjahjana, I. Pranoto, H. Muhammad and J. Naiborhu
}

\begin{abstract}
In this paper, we consider a numerical method for designing optimal control on Linear Quadratic Regulator (LQR) problem. In the optimal control design process through Pontryagin Maximum Principle (PMP), we obtain a system of differential equations in state and costate variables. This system lacks of initial condition on the adjoint variables, and this situation creates classic difficulty for solving optimal control problems. This paper proposes a constructive method to approximate the initial condition of the adjoint system.
\end{abstract}

\section{INTRODUCTION}

Let a linear control system be $\dot{\mathbf{x}}=A \mathbf{x}+B \mathbf{u}$. We want to drive the state $\mathbf{x}$ from a given initial condition to a given final condition in a finite time and minimize a cost functional. In solving this optimal control problem, Pontryagin's Maximum Principle is applied. This approach converses the optimal control problem into solving a Hamiltonian system consisting of a pair of linear differential equations. For solving this system of equations, one must solve a two point boundary value problem of the first equation. On the other hand, the second equation lacks of the initial condition. This is a classic problem in the optimal control design. In order to overcome this, we propose a constructive method to approximate the initial condition of the adjoint variable. This method is quite general in the sense that it can be applied to any kind of linear control systems.

There have been several different methods proposed by other researchers for overcoming the classical difficulty regarding the initial conditions of the adjoint variables. Scheeres et al [4] use generating functions in their work. In Twigg et

Received 25-02-2008, Accepted 14-10-2008.

2000 Mathematics Subject Classification: Primary 49J15; Secondary 35N05

Key words and Phrases: Optimal Control, Overdetermined Systems 
al [5], they use genetic algorithm for computing the optimal controls. Another approach is proposed by Kelly et al [2]. In their paper, they use polynomial spirals.

\section{LINEAR QUADRATIC REGULATOR PROBLEM}

We consider a Linear Quadratic Regular (LQR) problem as follows

$$
\dot{\mathbf{x}}=A \mathbf{x}+B \mathbf{u},
$$

where $\mathbf{x} \in \mathbf{R}^{n}$ and $\mathbf{u} \in \mathbf{R}^{m}$. The initial and final conditions are

$$
\mathbf{x}(0)=\mathbf{m}, \quad \mathbf{x}(T)=\mathbf{s} .
$$

The problem discussed here is to determine a control $\mathbf{u}$ driving the the state $\mathbf{x}$ from $\mathbf{m}$ to $\mathbf{s}$ in time $T$. At the same time, the control $\mathbf{u}$ must minimize the quadratic functional cost

$$
J=\frac{1}{2} \int_{0}^{T} \delta\|\mathbf{u}(t)\|^{2} d t,
$$

where $\delta$ is some positive constant. The integrand represents the cost or energy of the controls used. In (2), the symbol $\mathbf{x}(t)$ denotes the state at time $t$, the symbol $\mathbf{m}$ denotes the initial state, and $\mathbf{s}$ denotes the final state.

In order to make the discussion simpler, we consider a slightly different functional, namely it is the negative of the original $J$. So, we want to maximize a functional

$$
J^{\prime}=-\frac{1}{2} \int_{0}^{T} \delta\|\mathbf{u}(t)\|^{2} d t .
$$

Thus, instead of minimizing $J$, we will maximize $J^{\prime}$.

We assume that the control system is controllable. Because of the linearity of the system, the controllability implies strong controllability. This means that there exists a control $\mathbf{u}$ such that (2) is satisfied for however small the time $T$ is. Therefore, there is a $\mathbf{u}$ that makes $J^{\prime}$ finite. It implies that there exists a $\mathbf{u}$ maximizing $J^{\prime}$.

The Hamiltonian function derived from the optimal control problem above is

$$
H=\mathbf{p}^{T}(A \mathbf{x}+B \mathbf{u})-\frac{1}{2} \delta \mathbf{p}_{0}\|\mathbf{u}\|^{2}
$$

Using the Hamiltonian function above, one can derive the following Hamiltonian system

$$
\begin{aligned}
& \frac{\partial H}{\partial \mathbf{p}}=\dot{\mathbf{x}}=A \mathbf{x}+B \mathbf{u} \\
& \frac{\partial H}{\partial \mathbf{x}}=-\dot{\mathbf{p}}=A^{T} \mathbf{p}
\end{aligned}
$$


By the Pontryagin Maximum Principle, the extremal trajectory must satisfy

$$
\frac{\partial H}{\partial \mathbf{u}}=0=B^{T} \mathbf{p}-\delta p_{0} \mathbf{u}
$$

and $p_{0}$ must be a positive constant. One may consult Hocking [1], for more detailed explanation. We assume $p_{0}=1$. If one substitutes the control $\mathbf{u}$ from (5) into (3), we obtain a system of differential equations as follows

$$
\begin{aligned}
\dot{\mathbf{x}} & =A \mathbf{x}+\frac{1}{\delta p_{0}} B B^{T} \mathbf{p} \\
-\dot{\mathbf{p}} & =A^{T} \mathbf{p}
\end{aligned}
$$

Using (7), one obtains

$$
\mathbf{p}(t)=\exp \left(-A^{T} t\right) \mathbf{k}
$$

for some $\mathbf{k}$.

The system consisting a pair of differential equations (6-7) must be solved to find the optimal trajectory of $\mathbf{x}$. The first equation (6) has initial and final conditions that must be satisfied. However, the initial condition of the second equation (7) is not known. This is a classic difficulty in the optimal control theory.

\section{Steepest Descent Method}

Because the initial condition of the second equation (7) is not known, we create a method for constructing an approximation of the suitable initial condition.

If we let $\mathbf{p}(0)=\mathbf{q}$ for some $\mathbf{q} \in \mathbf{R}^{n}$, we obtain

$$
\mathbf{p}(t)=\exp \left(-A^{T} t\right) \mathbf{q}
$$

Next, if we use (8) in (6), we obtain

$$
\dot{\mathbf{x}}=A \mathbf{x}+\frac{1}{\delta} B B^{T} \exp \left(-A^{T} t\right) \mathbf{q}
$$

Therefore, the final state $\mathbf{x}(T)$ with $\mathbf{p}(0)=\mathbf{q}$ is

$$
\mathbf{x}(T ; \mathbf{q})=\exp (A T)\left(\mathbf{x}(0)+\frac{1}{\delta} \int_{0}^{T} \exp (-A t) B B^{T} \exp \left(-A^{T} t\right) d t \mathbf{q}\right)
$$

Of course, the trajectory $\mathbf{x}$ in general will not end at $\mathbf{s}$. In other words, in general $\mathbf{x}(T) \neq \mathbf{s}$.

To guess a suitable $\mathbf{q}$ that makes $\mathbf{x}$ start exactly from $\mathbf{m}$ and end exactly at $\mathbf{s}$ in time $T$ is not easy. Thus, we propose an algorithm for approximating a suitable q. 

functional

Instead of finding the precise $\mathbf{q}$ above, we want to find $\mathbf{q}$ that minimizes the

$$
F(\mathbf{q})=\|\mathbf{x}(T)-\mathbf{s}\|^{2}
$$

where $\mathbf{x}(T)$ is the evaluation of $\mathbf{x}$ at time $T$ and $(\mathbf{x}, \mathbf{p})$ is the solution of differential equation system (6)- (7) with initial conditions $(\mathbf{x}(0), \mathbf{p}(0))=(\mathbf{m}, \mathbf{q})$. We minimize the functional $F$ by employing the Steepest Descent Method.

The algorithm can be described as follows. First, we pick any positive numbers $\epsilon, \alpha$ and any vector $\mathbf{q}^{0} \in \mathbf{R}^{n}$. Using these and the initial condition $\mathbf{x}(0)=\mathbf{m}$, one can solve the initial value problem

$$
\begin{aligned}
\dot{\mathbf{x}} & =A \mathbf{x}+\frac{1}{\delta p_{0}} B B^{T} \mathbf{p} \\
-\dot{\mathbf{p}} & =A^{T} \mathbf{p}
\end{aligned}
$$

where $(\mathbf{x}(0), \mathbf{p}(0))=\left(\mathbf{m}, \mathbf{q}^{0}\right)$. In particular, one can compute the final state $\mathbf{x}(T)$ for this particular $\mathbf{q}^{0}$. Moreover, using this $\mathbf{x}(T)$, one can compute (9) to determine the scalar value $F\left(\mathbf{q}^{0}\right)$. Next, we want to find a new $\mathbf{q}^{1}$ that will make $F\left(\mathbf{q}^{1}\right)$ smaller than $F\left(\mathbf{q}^{0}\right)$.

The partial derivatives of $F\left(p_{1}, p_{2}, \cdots, p_{n}\right)$ with respect to $p_{i}$ at $\mathbf{q}^{0}$ is approximated by

$$
\begin{aligned}
& \frac{\partial F}{\partial p_{1}}\left(\mathbf{q}^{0}\right) \approx D_{1}=\frac{F\left(\mathbf{q}^{0}+\epsilon(1,0,0, \cdots, 0,0)\right)-F\left(\mathbf{q}^{0}\right)}{\epsilon} \\
& \frac{\partial F}{\partial p_{2}}\left(\mathbf{q}^{0}\right) \approx D_{2}=\frac{F\left(\mathbf{q}^{0}+\epsilon(0,1,0, \cdots, 0,0)\right)-F\left(\mathbf{q}^{0}\right)}{\epsilon} \\
& \frac{\partial F}{\partial p_{n}}\left(\mathbf{q}^{0}\right) \approx D_{n}=\frac{F\left(\mathbf{q}^{0}+\epsilon(0,0,0, \cdots, 0,1)\right)-F\left(\mathbf{q}^{0}\right)}{\epsilon} .
\end{aligned}
$$

Thus, the gradient of $F$ at $\mathbf{q}^{0}$ is approximated by

$$
\nabla F\left(\mathbf{q}^{0}\right)=\left(\frac{\partial F}{\partial p_{1}}\left(\mathbf{q}^{0}\right), \frac{\partial F}{\partial p_{2}}\left(\mathbf{q}^{0}\right), \cdots, \frac{\partial F}{\partial p_{n}}\left(\mathbf{q}^{0}\right)\right) \approx\left(D_{1}, D_{2}, \cdots, D_{n}\right)
$$

Next, we set

$$
\begin{aligned}
& E_{1}=F\left(\mathbf{q}^{0}-\alpha\left(D_{1}, D_{2}, \cdots, D_{n}\right)\right) \\
& E_{2}=F\left(\mathbf{q}^{0}-\left(\frac{\alpha}{2}\right)\left(D_{1}, D_{2}, \cdots, D_{n}\right)\right) .
\end{aligned}
$$

If $E_{1}<E_{2}$, we let $\mathbf{q}^{1}=\mathbf{q}^{0}-\alpha\left(D_{1}, D_{2}, \cdots, D_{n}\right)$. Otherwise, we let $\mathbf{q}^{1}=\mathbf{q}^{0}-$ $\frac{\alpha}{2}\left(D_{1}, D_{2}, \cdots, D_{n}\right)$ and replace $\epsilon$ as $\frac{\epsilon}{2}$ and $\alpha$ as $\frac{\alpha}{2}$. We can start again using this $\mathbf{q}^{1}$ in place of $\mathbf{q}^{0}$ to determine $\mathbf{q}^{2}$. If we continue this process, we will obtain a vector sequence $\left\{\mathbf{q}^{m}\right\}_{m=0}^{\infty}$. 
The next task is to verify the convergence of the proposed algorithm. First, we prove the following theorem

Theorem 3.1. The functional $F$ defined in (9) is strictly convex as a function of q.

Proof. Suppose $\mathbf{r}=\exp (A T) \mathbf{x}(0)$ and

$$
Q=\exp (A T) \frac{1}{\delta} \int_{0}^{T} \exp (-A t) B B^{T} \exp \left(-A^{T} t\right) d t .
$$

So, the final state $\mathbf{x}(T)$ with the initial condition of the adjoint variable equals $\mathbf{q}$ is $\mathbf{x}(T ; \mathbf{q})=\mathbf{r}+Q \mathbf{q}$. Thus, it is an affine function with respect to $\mathbf{q}$.

Let $\mathbf{q}$ be $\lambda \mathbf{v}+(1-\lambda) \mathbf{w}$, where $\mathbf{v}, \mathbf{w} \in \mathbf{R}^{2}, \mathbf{v} \neq \mathbf{w}$, and $\lambda$ satisfies $0<\lambda<1$. Thus,

$$
\begin{aligned}
(F \circ \mathbf{x})(\lambda \mathbf{v}+(1-\lambda) \mathbf{w}) & =F(\mathbf{x}(\lambda \mathbf{v}+(1-\lambda) \mathbf{w})) \\
& =F(\mathbf{r}+Q(\lambda \mathbf{v}+(1-\lambda) \mathbf{w})) \\
& =\|\mathbf{r}+Q(\lambda \mathbf{v}+(1-\lambda) \mathbf{w})-\mathbf{s}\|^{2} \\
& =\| \mathbf{r}+Q \lambda \mathbf{v}+Q(1-\lambda) \mathbf{w})-\mathbf{s} \|^{2} \\
& =\| \mathbf{r}+\lambda Q \mathbf{v}+(1-\lambda) Q \mathbf{w})-\mathbf{s} \|^{2} \\
& <\lambda\|\mathbf{r}+Q \mathbf{v}-\mathbf{s}\|^{2}+(1-\lambda)\|\mathbf{r}+Q \mathbf{w}-\mathbf{s}\|^{2} \\
& =\lambda F(\mathbf{r}+Q \mathbf{v})+(1-\lambda) F(\mathbf{r}+Q \mathbf{w}) \\
& =\lambda F(\mathbf{x}(v))+(1-\lambda) F(\mathbf{x}(\mathbf{w})) \\
& =\lambda F \circ \mathbf{x}(\mathbf{v})+(1-\lambda) F \circ \mathbf{x}(\mathbf{w})
\end{aligned}
$$

Therefore,

$$
(F \circ \mathbf{x})(\lambda \mathbf{v}+(1-\lambda) \mathbf{w})<\lambda F \circ \mathbf{x}(\mathbf{v})+(1-\lambda) F \circ \mathbf{x}(\mathbf{w})
$$

and we conclude that $F$ is strictly convex with respect to $\mathbf{q}$.

Next, using any initial condition of $F$, we need the guarantee that the steepest descent sequence is convergent to the unique global minimizer of $F$. So, the following lemma is needed.

Lemma 2.1. If $F$ is a strictly convex, coercive function with continuous first partial derivatives on $\mathbf{R}^{n}$, then for any initial point $\mathbf{x}(0)$, the steepest descent sequence with initial point $\mathbf{x}(0)$ converges to the unique global minimizer of $F$.

Interested readers may find the proof in [3].

Now, we are ready to state the main result of this paper in the next theorem. 
Theorem 3.2. The sequence $\left\{\mathbf{q}^{m}\right\}$ converges to a suitable initial condition $\mathbf{p}$ that makes the final state $\mathbf{x}(T)$ equal $\mathbf{s}$.

Proof.By Theorem 3.1, the functional $F \circ \mathbf{x}$ is strictly convex with respect to $\mathbf{q}$. Obviously, $F \circ \mathbf{x}$ is a coercive function, again with respect to $\mathbf{q}$. By Lemma 3.1, we conclude that the steepest descent sequence $\left\{\mathbf{q}^{m}\right\}$ converges to the global minimizer of $F \circ \mathbf{x}$. This means that the sequence $\left\{\mathbf{x}\left(T ; \mathbf{q}^{m}\right)\right\}$ converges to $\mathbf{s}$ as $m$ goes to infinity. At the same time, the sequence $\left\{\mathbf{q}^{m}\right\}$ converges to a suitable initial condition of the adjoint variable that makes the state $\mathbf{x}$ reach $\mathbf{s}$ at time $T$.

In the next section, we give an example to validate the proposed method. It shows that the numerical results obtained by the proposed method are close to the theoretical solution.

\section{AN EXAMPLE}

Consider the following linear system

$$
\left(\begin{array}{l}
\dot{y_{1}} \\
\dot{y_{2}}
\end{array}\right)=\left(\begin{array}{cc}
-1 & 1 \\
0 & -1
\end{array}\right)\left(\begin{array}{l}
y_{1} \\
y_{2}
\end{array}\right)+\left(\begin{array}{l}
2 \\
3
\end{array}\right) v,
$$

where the initial and final conditions are given as follows

$$
\left(\begin{array}{l}
y_{1}(0) \\
y_{2}(0)
\end{array}\right)=\left(\begin{array}{l}
1 \\
2
\end{array}\right) \quad\left(\begin{array}{l}
y_{1}(1) \\
y_{2}(1)
\end{array}\right)=\left(\begin{array}{l}
3 / e \\
2 / e
\end{array}\right) \approx\left(\begin{array}{c}
1.103638324 \\
0.7357588824
\end{array}\right) .
$$

The cost functional that must be maximized is

$$
J=-\frac{1}{2} \int_{0}^{1} v^{2} d t
$$

When $v \equiv 0$, the final state at $t=1$ is

$$
\left(\begin{array}{l}
y_{1}(1) \\
y_{2}(1)
\end{array}\right)=e^{-1}\left(\begin{array}{l}
3 \\
2
\end{array}\right) \text {. }
$$

Thus, $v \equiv 0$ must be the exact optimal control. The Hamiltonian function of the above system is

$$
H=p_{1}\left(-y_{1}+y_{2}+2 v_{1}\right)+p_{2}\left(-y_{2}+3 v_{1}\right)-\frac{1}{2} p_{0} v^{2}
$$

The Hamiltonian system is

$$
\begin{aligned}
& \frac{\partial H}{\partial p_{1}}=\dot{y_{1}}=-y_{1}+y_{2}+2 v \\
& \frac{\partial H}{\partial p_{2}}=\dot{y_{2}}=-y_{2}+3 v \\
& \frac{\partial H}{\partial y_{1}}=-\dot{p_{1}}=-p_{1} \\
& \frac{\partial H}{\partial y_{2}}=-\dot{p_{2}}=p_{1}-p_{2}
\end{aligned}
$$


By the Pontryagin Maximum Principle, a necessary condition that makes the system optimal is

$$
\frac{\partial H}{\partial v}=0=2 p_{1}+3 p_{2}-p_{0} v .
$$

Thus, one obtains

$$
v=\frac{2 p_{1}+3 p_{2}}{p_{0}} .
$$

So, now we have to solve the differential equation system as follows

$$
\begin{aligned}
& \dot{y_{1}}=-y_{1}+y_{2}+\frac{4 p_{1}+6 p_{2}}{p_{0}} \\
& \dot{y_{2}}=-y_{2}+\frac{6 p_{1}+9 p_{2}}{p_{0}} \\
& -\dot{p_{1}}=-p_{1} \\
& -\dot{p_{2}}=p_{1}-p_{2}
\end{aligned}
$$

Since the exact optimal control of the above case is the zero control, it means that the exact values of $p_{1}(0)$ and $p_{2}(0)$ must be zero. We show that the proposed numerical method approaches these values as well.

We apply the proposed method to the above system. First, we let the error tolerance be $10^{-3}$. If we set an initial guess

$$
\left(\begin{array}{l}
p_{1}(0) \\
p_{2}(0)
\end{array}\right)=\left(\begin{array}{l}
1 \\
2
\end{array}\right)
$$

and $\epsilon=\alpha=1$, after 1430 iterations, we obtain

$$
\left(\begin{array}{l}
p_{1}(0) \\
p_{2}(0)
\end{array}\right)=\left(\begin{array}{c}
0.061461356213579498597 \\
-0.0028077374883373888168
\end{array}\right) .
$$

Using this $\left(\begin{array}{l}p_{1}(0) \\ p_{2}(0)\end{array}\right)$, one obtains

$$
\left(\begin{array}{l}
y_{1}(1) \\
y_{2}(1)
\end{array}\right)=\left(\begin{array}{c}
1.1251491341904354839 \\
0.71265956095991731193
\end{array}\right) .
$$

This is close to the terminal condition in (11). Second, we use a smaller error tolerance. Let the error tolerance be $10^{-6}$. Using the same initial guess, after 1736 iterations, we obtain

$$
\left(\begin{array}{l}
p_{1}(0) \\
p_{2}(0)
\end{array}\right)=\left(\begin{array}{c}
0.0019435296484999768101 \\
-0.000088768635445718336227
\end{array}\right) .
$$

If we plug this $\left(\begin{array}{c}p_{1}(0) \\ p_{2}(0)\end{array}\right)$ into (12) one obtains

$$
\left(\begin{array}{l}
y_{1}(1) \\
y_{2}(1)
\end{array}\right)=\left(\begin{array}{c}
1.1043188037904674930 \\
0.73502860475158591577
\end{array}\right) \text {. }
$$


This is closer to the terminal condition (11). Thus, we see that the smaller the error tolerance, the closer the $\left(\begin{array}{l}p_{1}(0) \\ p_{2}(0)\end{array}\right)$ to $\left(\begin{array}{l}0 \\ 0\end{array}\right)$. These results verify that the proposed method and theory lead to the exact optimal control.

\section{REFERENCES}

1. L.M. Hocking, Optimal Control, Oxford University Press, 1991.

2. A. Kelly And B. NAGy, "Reactive Non holonomic Trajectory Generation via Parametric Optimal Control", The International Journal of Robotics Research (2003), 583-601.

3. A.L. Peressini, F.E. Sullivan, And J.J. Uhl, The Mathematics of Nonlinear Programming, Springer-Verlag, 1988.

4. D.J. Scheeres, C. Park and A. Guibout, "Solving Optimal Control Problem with Generating Functions", Paper presented at The Astrodynamics Specialist Conferences, Big Sky, Montana, (2003).

5. S. Twigg, A. Calise and E. Johnson, "Trajectory Optimization for Multiple Vehicles Using a reduced Order Formulation", Paper presented at Infotech at Aerospace, Arlington, Virginia, (2005).

H. TJahjana: Department of Mathematics, Institut Teknologi Bandung, Bandung 40132, Indonesia.

Permanent Address: Department of Mathematics, UNDIP, Semarang, Indonesia

E-mail: heru_tjahjana@students.itb.ac.id.

I. Pranoto: Department of Mathematics, Institut Teknologi Bandung, Bandung 40132, Indonesia.

E-mail: pranoto@math.itb.ac.id.

H. Muhammad: Department of Aeronautics and Aerospace Engineering, Institut Teknologi Bandung, Bandung 40132, Indonesia.

E-mail: harmad@ae.itb.ac.id.

J. NAiborhu: Department of Mathematics, Institut Teknologi Bandung, Bandung 40132, Indonesia.

E-mail: janson@math.itb.ac.id. 